\title{
Effect of Bepridil in Atrial Fibrillation Inducibility Facilitated by Vagal Nerve Stimulation of the Atrial Action Potential Duration -
}

Kenichi Iijima, MD; Masaomi Chinushi, MD*; Daisuke Izumi, MD; Shizue Ahara*; Hiroshi Furushima, MD; Satoru Komura, MD; Yukio Hosaka, MD; Akiko Sanada, MD; Akinori Sato, MD; Yoshifusa Aizawa, MD

\begin{abstract}
Background: Because bepridil blocks multiple myocardial ionic channels, including the muscarinic acetylcholine receptor-operated potassium current (IKAch), bepridil is expected to suppress atrial fibrillation (AF) mediated by vagal nerve stimulation (VNS).

Methods and Results: The therapeutic effects of bepridil were studied with a special focus on heart rate variability (HRV) in a canine model of AF. During VNS, AF was induced in 9 of 9 experiments before, vs 3 of 9 experiments after administration of bepridil $(P<0.01)$. During $350 \mathrm{~ms}$ atrial pacing, VNS shortened the right and left atrial monophasic action potentials at $90 \%$ repolarization (MAP90) by $-31 \pm 8 \%$ and $-22 \pm 12 \%$, respectively, vs $-10 \pm 13 \%$ and $-6 \pm 8 \%$, respectively, after bepridil $(P<0.01, N=9)$. Bepridil prolonged the sinus cycle length, although it had no significant effect on the conduction time measured at $300 \mathrm{~ms}$ pacing. Statistically insignificant change was observed in the VNS-induced slowing of the sinus cycle length and in the VNS-induced increase in high frequency amplitude of HRV before $(1.2 \pm 0.7$ to $5.3 \pm 4.0 \mathrm{~ms})$ vs after $(1.7 \pm 0.8$ to $5.4 \pm 2.3 \mathrm{~ms})$ bepridil administration.
\end{abstract}

Conclusions: Bepridil prevented the VNS-induced shortening of atrial MAP90 and suppressed the inducibility of AF during VNS in two-thirds of the experiments. As far as this study shows, it may be possible that inhibition of IKAch played a part in this antifibrillatory effect. (Circ $J$ 2010; 74: 895-902)

Key Words: Atrial fibrillation; Bepridil; Muscarinic acetylcholine receptor-operated potassium current; Vagal nerve activity

B epridil, a pharmaceutical that inhibits multiple myocardial ionic currents, including calcium, sodium and several potassium currents, ${ }^{1-3}$ is used to treat ventricular tachyarrhythmias and atrial fibrillation (AF). ${ }^{4,5}$ Although bepridil has been found to be highly effective in the treatment of $\mathrm{AF},{ }^{4-7}$ its electrophysiological properties are not fully understood.

In AF associated with structural heart disease, an arrhythmogenic substrate is created by atrial enlargement and by structural and electrophysiological remodeling of the myocardium. On the other hand, triggered activity originating from the pulmonary veins, and reentrant activation around their orifices, are important mechanisms of lone AF. ${ }^{8,9}$ In both instances, enhanced vagal nerve activity is proarrhythmic and facilitates the development of AF.

Because bepridil inhibits the muscarinic acetylcholine receptor-operated potassium current (IKAch), ${ }^{10}$ it is expected to be effective in the treatment of AF associated with vagal activity, by inhibiting the acetylcholine-induced increase in IKAch in the atrium. We tested this hypothesis in a previously described canine model of AF facilitated by vagal nerve stimulation (VNS). ${ }^{11-13}$ Besides the inducibility of AF and the electrophysiological characteristics of the atrium, we studied the antiarrhythmic effects of bepridil in AF associated with enhanced vagal nerve activity, and measured autonomic nervous activity using heart rate variability (HRV).

\section{Methods}

\section{Animal Preparation}

This study was approved by the Animal Studies Subcommittee of our Institutional Review Board, and was in compliance with the guidelines issued by the United States National Institutes of Health for the Care and Use of Laboratory Animals.

Received September 27, 2009; revised manuscript received January 15, 2010; accepted January 31, 2010; released online April 3, 2010

Time for primary review: 29 days

The First Department of Internal Medicine, *School of Health Science, Niigata University School of Medicine, Niigata, Japan

Mailing address: Masaomi Chinushi, MD, School of Health Science, Niigata University School of Medicine, 2-746 Asahimachi, Chuou-ku,

Niigata 951-8510, Japan. E-mail: masaomi@clg.niigata-u.ac.jp

ISSN-1346-9843 doi:10.1253/circj.CJ-09-0716

All rights are reserved to the Japanese Circulation Society. For permissions, please e-mail: cj@j-circ.or.jp 


\section{Surgical Procedures}

The experiments were performed in 9 beagles each weighing $9.5-10.3 \mathrm{~kg}$, anesthetized with a $17.5-\mathrm{mg} / \mathrm{kg}$ i.v. bolus of sodium thiopental, intubated and artificially ventilated. To minimize the effect of anesthetics on the autonomic nervous system, a $3.0-5.0 \mathrm{mg} \cdot \mathrm{kg}^{-1} \cdot \mathrm{h}^{-1}$ dose of thiopental was infused to maintain a shallow and constant sedation. Catheters were inserted into the femoral vein to administer fluids and drugs, and into the femoral artery to monitor arterial blood pressure. Leads I, II, III and aVF of the surface electrocardiogram and blood pressure were continuously monitored. The core temperature was kept at $37^{\circ} \mathrm{C}$ with a thermostatically controlled thermal blanket and infrared heating system. To stimulate the vagal nerve, two $75-\mu \mathrm{m}$ silver wire electrodes were inserted into the right cervical vagal trunk, and square pulses of $0.5-2.0 \mathrm{~V}$ in strength and $0.3 \mathrm{~ms}$ in duration were delivered at a frequency of $20 \mathrm{~Hz} \cdot{ }^{14,15}$ The heart was exposed via midline sternotomy. A model 1675P Franz catheter (Boston Scientific, Natick, MA, USA) was inserted into the left pulmonary vein and advanced retrogradely to the lateral side of the left atrium near the mitral valve, to record left atrial (LA) monophasic action potential (MAP) and local bipolar electrogram. Another Franz catheter was advanced to the right atrial (RA) free wall from the right femoral vein, to record the RA electrogram. Two pairs of silver wire hook electrodes were inserted into the RA appendage and right ventricle, respectively, for electrical stimulation. Pacing pulses, $2.0 \mathrm{~ms}$ in duration at twice the diastolic threshold, were delivered by a programmable cardiac stimulator. Upon completion of the experiments, the animals were sacrificed by electrical induction of ventricular fibrillation under deep general anesthesia.

\section{Data Acquisition and Analysis}

The signals from each intracardiac catheter were recorded on a MCS-5500 polygraph (Fukuda Densi Co Ltd, Tokyo, Japan) at a paper speed of $100 \mathrm{~mm} / \mathrm{s}$, using a filter setting of 0.05 $500 \mathrm{~Hz}$ for MAP recordings and $50-500 \mathrm{~Hz}$ for bipolar electrograms. The MAP duration at $90 \%$ repolarization (MAP90) at a basic pacing length of $350 \mathrm{~ms}$ was measured as the index action potential duration. During the atrial pacing at $350 \mathrm{~ms}$, atrioventricular conduction block was observed after administration of bepridil and during the application of VNS before administration of bepridil. However, we did not measure the pacing cycle length showing Wenckebach atrioventricular conduction in order to complete the experimental protocol within a reasonably short interval to best measure the pharmacological effects of bepridil. The interatrial conduction time (CT-ia) was measured between the stimulus artifact delivered in the RA appendage and the local LA activation at a pacing cycle length of $300 \mathrm{~ms}$. The effective refractory period (ERP) of the right atrium was measured using standard techniques, with trains of 10 regular stimuli (S1) at a pacing cycle length of $300 \mathrm{~ms}$, and extrastimuli (S2) delivered at a coupling interval (S1S2) shortened in steps of $2 \mathrm{~ms}$. ERP-RA was defined as the longest S1S2 interval at which S2 failed to capture the atrium. AF was induced, using up to 2 extrastimuli as a basic pacing cycle length of $300 \mathrm{~ms}$ or rapid pacing up to $200 \mathrm{~ms}$.

\section{Measurements of HRV}

Body surface electrocardiogram recordings were digitized at a sampling rate of $1,000 \mathrm{~Hz}$ by an ADX-002 analog-to-digital converter (ADTEC Inc, Tokyo, Japan) and stored in a personal computer. The cardiac cycle length was measured using
GM-View II R-wave detection software (Signalysis Ltd, Saitama, Japan). The MemCalc method ${ }^{16-18}$ was used to calculate the high-frequency $(\mathrm{HF} ; 0.15-0.4 \mathrm{~Hz})$ and low-frequency (LF; $0.04-0.15 \mathrm{~Hz}$ ) components of HRV, using continuous 90-s samples of RR intervals, using MemCalc, Version 2.0, software (Suwa Trust Co, Tokyo, Japan). MemCalc combines a maximum entropy method for the spectral analysis and a non-linear least squares method for the fitting analysis, allowing the analysis of both LF and HF components over a minimum sampling interval of $30 \mathrm{~s} .{ }^{16-18}$ In this study, the LF and HF components were expressed as their amplitude $\left[\mathrm{LF}-\mathrm{amp}=(2 \mathrm{LF} \text { component })^{1 / 2}\right.$ and HF-amp $=(2 \mathrm{HF}$ component $)^{1 / 2}$ and the LF/HF ratio was calculated. ${ }^{11}$

\section{Study Protocol and Data Collection}

The experimental protocol was completed within $2 \mathrm{~h}$ after the surgical preparation and instrumentation of the animals. In this study, we focused on the inducibility of AF with or without application of VNS as a parameter of the antiarrhythmic effect of bepridil for AF.

Before Administration of Depridil Completion of the following protocols of A-C took approximately 60-90 min.

(A) First, MAP90 in both atria, CT-ia and ERP-RA were measured. Then, programmed electrical stimulation was applied to examine the inducibility of AF.

(B) Second, right VNS was gradually increased from $0.5 \mathrm{~V}$ in steps of $0.1 \mathrm{~V}$, and the lowest voltage that induced AF for $>30$ s (VNS-min.V) was obtained. ${ }^{11}$ Once AF was induced and sustained, VNS was discontinued within $45 \mathrm{~s}$ to avoid the development of electrical remodeling in the atrium. ${ }^{19}$ The VNS-min.V used in this study was $1.2 \pm 0.5 \mathrm{~V}(\mathrm{~N}=9)$. During the application of VNS-min.V, MAP90, CT-ia and ERP-RA were again measured. Demand back-up ventricular pacing at a cycle length of $1,000 \mathrm{~ms}$ was used to prevent profound bradycardia during all episodes of VNS.

(C) Third, RR intervals were recorded for analysis of HRV during spontaneous rhythm, in absence and during VNS-min.V. If the sinus rate fell below that of junctional escape during VNS-min.V, the output of VNS was slightly decreased until reemergence of sinus rhythm, to allow the sampling of RR intervals for analysis of HRV during VNS.

After Bepridil Administration Completion of the following protocols of $\mathbf{A}-\mathbf{B}$ was within $30 \mathrm{~min}$.

Bepridil, $4 \mathrm{mg} / \mathrm{kg}$, was dissolved into sterile saline and infused intravenously over $5 \mathrm{~min}$.

(A) First, $5 \mathrm{~min}$ after the administration of bepridil was completed, the RR intervals were sampled during sinus rhythm, before and during VNS, at an output identical to that delivered before drug administration.

(B) Second, MAP90, CT-ia and ERP-RA were measured, and the inducibility of AF was examined, before and during the application of VNS-min.V. If AF was induced, the mean F-F intervals during application of VNS-min.V before and after administration of bepridil were compared.

\section{Statistical Analysis}

Data are presented as means \pm SD. The electrophysiological measurements were compared using a paired t-test. The inducibility of AF was analyzed by Fisher's exact probability method. The variables of HRV were compared using Wilcoxon's signed-rank sum test. Differences in the hemodynamic and electrophysiological data among the 3 periods, ie, beginning, middle (approximately $15 \mathrm{~min}$ after starting the bepridil protocol) and completion of the experimental protocol of bepridil, were examined with repeated measures 
A

Before bepridil

Single extra stimulus

$\left(\mathrm{S}_{1} \mathrm{~S}_{1} / \mathrm{S}_{1} \mathrm{~S}_{2}=300 / 64\right)$

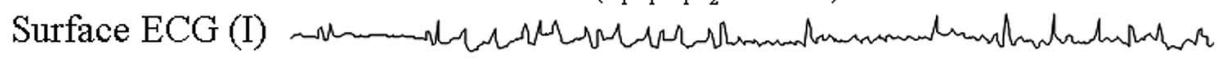

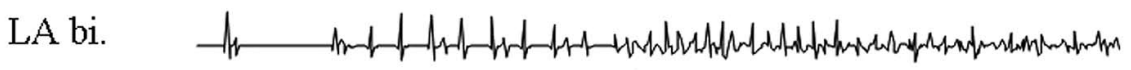

LA-MAP

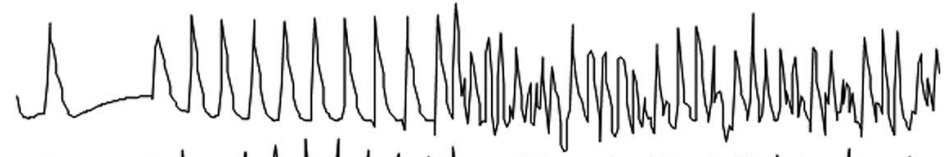

RA bi.

RA-MAP

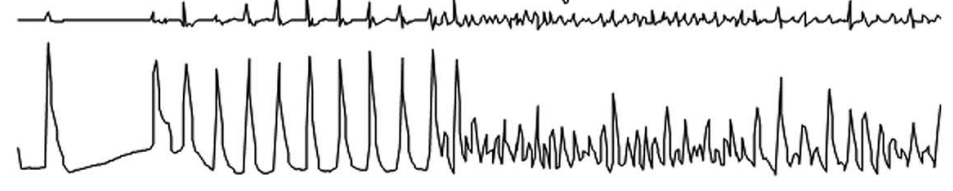

After bepridil

Double extra stimuli

$\left(\mathrm{S}_{1} \mathrm{~S}_{1} / \mathrm{S}_{1} \mathrm{~S}_{2} / \mathrm{S}_{2} \mathrm{~S}_{3}=300 / 160 / 110\right)$

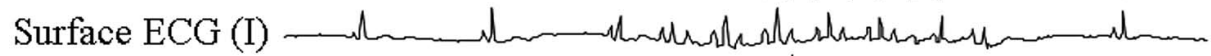

LA bi.

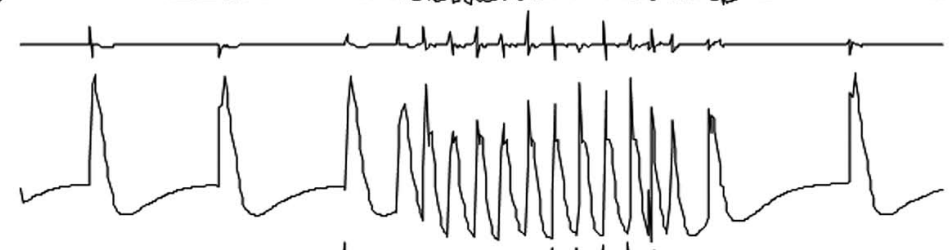

LA-MAP

RA bi.

RA-MAP

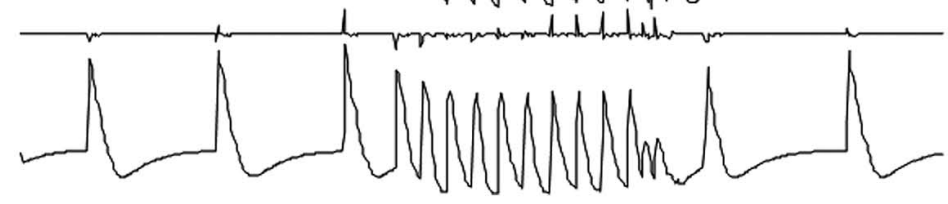

B

\section{Before bepridil}

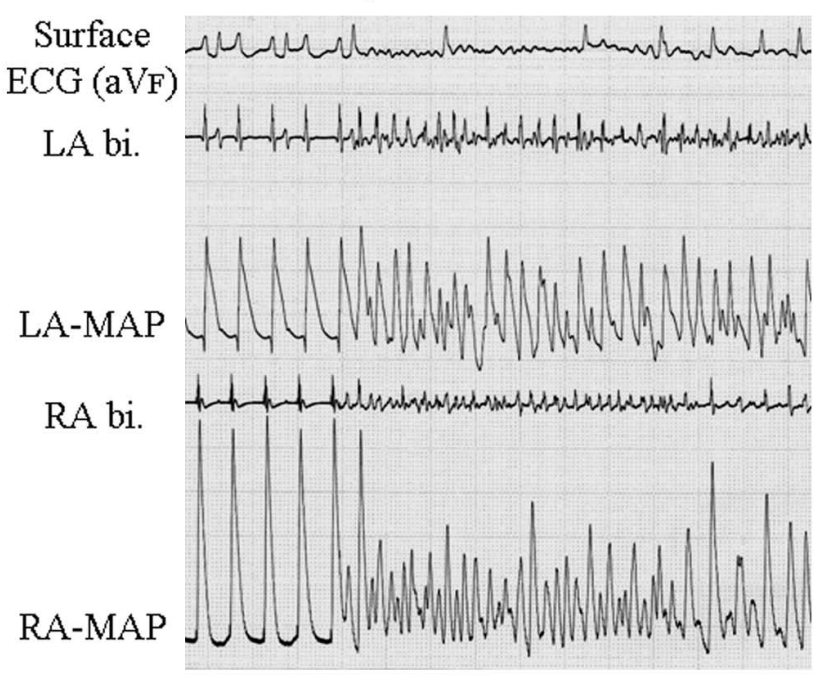

\section{After bepridil}

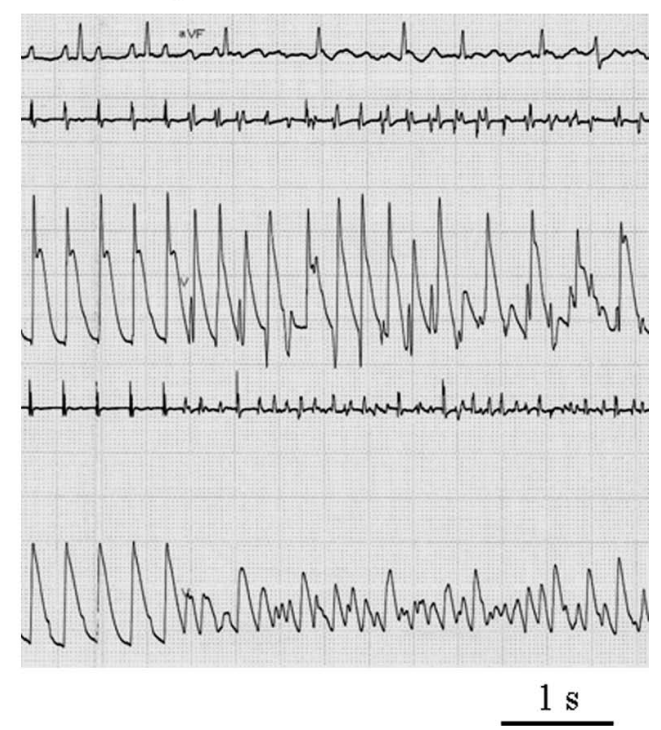

Figure 1. (A) Induction of atrial fibrillation (AF) by programmed electrical stimulation. Electrical stimuli were delivered during vagal nerve stimulation (VNS-min.V). Before administration of bepridil (Upper), sustained AF was induced in all 9 experiments. After administration of bepridil, AF became non-inducible during the application of VNS-min.V in 6 of the 9 experiments (Lower). (B) Prolongation of F-F interval after administration of bepridil. Before administration of bepridil, the mean F-F interval of induced AF was $91 \mathrm{~ms}$ (Left). In this experiment, AF was again induced after the administration of bepridil. However, the mean F-F interval was lengthened to $158 \mathrm{~ms}$ (Right). Surface ECG (I), lead I of surface electrocardiogram; LA bi, left atrial bipolar electrogram; LA-MAP, left atrial monophasic action potential; RA bi, right atrial bipolar electrogram; RA-MAP, right atrial monophasic action potential; $S_{1}$, basic pacing train; $S_{2}$ and $S_{3}$, second and third premature stimuli; Surface ECG $\left(a V_{F}\right)$, lead aVF of surface electrocardiogram. 


\begin{tabular}{|c|c|c|c|c|}
\hline & \multicolumn{2}{|c|}{ Before bepridil $(n=9)$} & \multicolumn{2}{|c|}{ After bepridil $(n=9)$} \\
\hline & VNS off & VNS on & VNS off & VNS on \\
\hline \multirow{2}{*}{ RR interval (ms) } & $523 \pm 104$ & $735 \pm 190^{\dagger}$ & $725 \pm 97$ & $950 \pm 166^{\dagger}$ \\
\hline & \multicolumn{2}{|c|}{$(+42 \pm 30 \%)$} & \multicolumn{2}{|c|}{$(+32 \pm 22 \%)$} \\
\hline \multirow{2}{*}{ ERP-RA (CL $300 \mathrm{~ms}, \mathrm{~ms})$} & $133 \pm 23$ & $74 \pm 13^{\dagger}$ & $180 \pm 19$ & $150 \pm 21^{\dagger}$ \\
\hline & \multicolumn{2}{|c|}{$(-43 \pm 10 \%)$} & \multicolumn{2}{|c|}{$(-16 \pm 11 \%)$} \\
\hline \multirow{2}{*}{ RA-MAP90 (CL $350 \mathrm{~ms}, \mathrm{~ms})$} & $162 \pm 22$ & $110 \pm 9^{\dagger}$ & $184 \pm 33$ & $166 \pm 39$ \\
\hline & \multicolumn{2}{|c|}{$(-31 \pm 8 \%)$} & \multicolumn{2}{|c|}{$(-10 \pm 13 \%)$} \\
\hline \multirow{2}{*}{ LA-MAP90 (CL $350 \mathrm{~ms}, \mathrm{~ms})$} & $169 \pm 19$ & $133 \pm 24^{\dagger}$ & $190 \pm 15$ & $179 \pm 25$ \\
\hline & \multicolumn{2}{|c|}{$(-22 \pm 12 \%)$} & \multicolumn{2}{|c|}{$(-6 \pm 8 \%)$} \\
\hline CT-ia (CL 300 ms, ms) & $54 \pm 7$ & $54 \pm 8$ & \multicolumn{2}{|c|}{$( \pm 0 \%)$} \\
\hline AF inducibility & $\begin{array}{c}0 / 9 \\
(0 \%)\end{array}$ & $\begin{array}{c}9 / 9 \\
(100 \%)\end{array}$ & $\begin{array}{c}0 / 9 \\
(0 \%)\end{array}$ & $\begin{array}{c}3 / 9^{*} \\
(33 \%)\end{array}$ \\
\hline
\end{tabular}

${ }^{\dagger} \mathrm{P}<0.05$ vs control; ${ }^{*} \mathrm{P}<0.05$ vs before drug administration with VNS.

VNS, vagal nerve stimulation; ERP-RA, effective refractory period of right atrium; CL, basic cycle length; RA-MAP90, the monophasic action potential duration at $90 \%$ repolarization (MAP90) in the right atrium; LA-MAP90, MAP90 in the left atrium; CT-ia, intra-atrial conduction time; AF, atrial fibrillation.

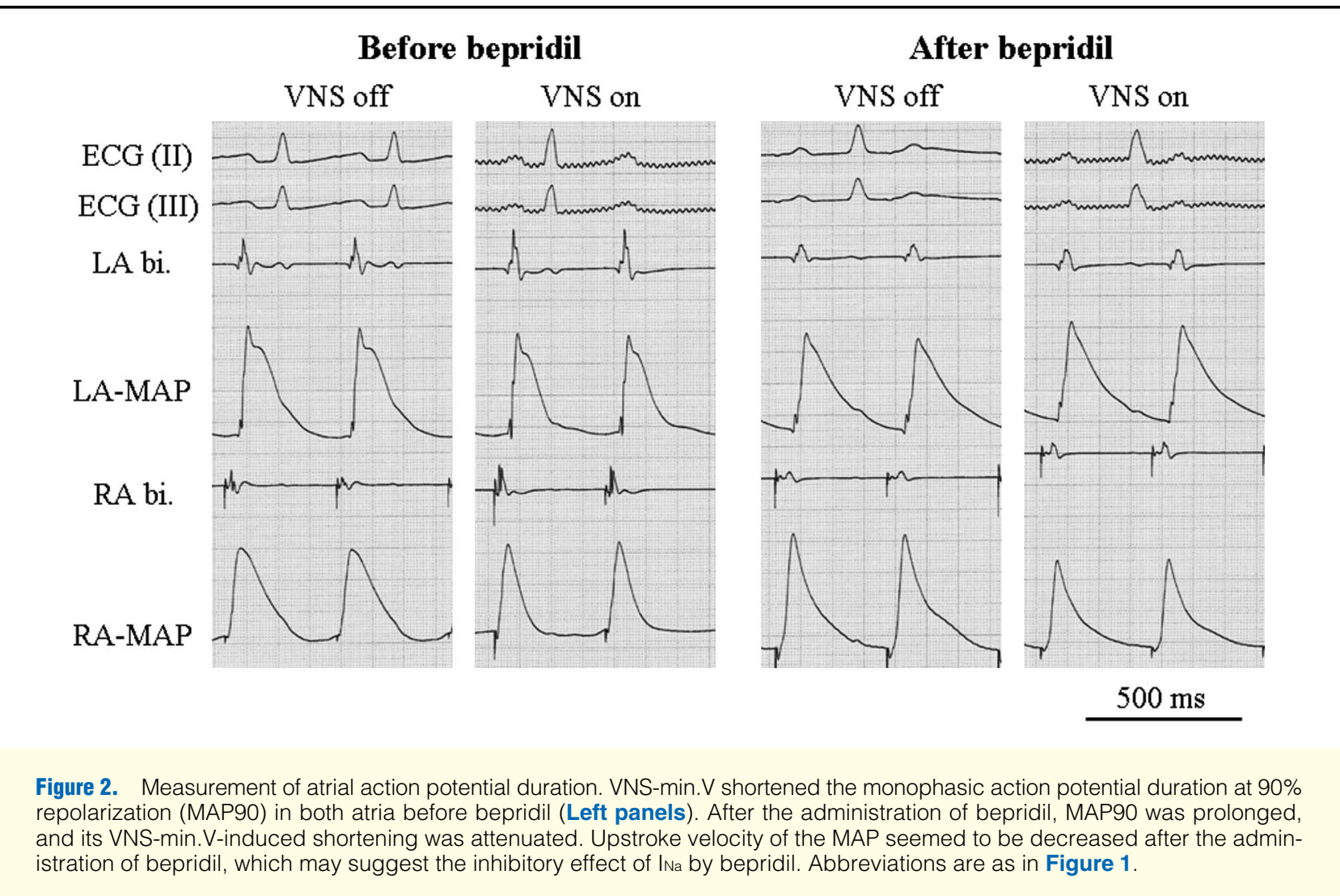

analysis of variance (ANOVA) with Bonferroni's correction. A P-value of $<0.05$ was considered statistically significant.

\section{Results}

\section{Effects of Bepridil on Heart Rate, Blood Pressure and Electrocardiogram}

Before the administration of bepridil, the mean sinus cycle length was $523 \pm 104 \mathrm{~ms}(\mathrm{~N}=9)$, and the mean systolic/diastolic blood pressure was $120 \pm 20 / 64 \pm 14 \mathrm{mmHg}(\mathrm{N}=9)$. Bepridil prolonged the sinus cycle length to $725 \pm 97 \mathrm{~ms}(\mathrm{P}<0.01, \mathrm{~N}=9)$ and decreased the systolic/diastolic blood pressure to $101 \pm$ $18 / 55 \pm 8 \mathrm{mmHg}(\mathrm{P}<0.05, \mathrm{~N}=9)$. The PQ interval lengthened from $110 \pm 12$ to $131 \pm 7 \mathrm{~ms}(\mathrm{P}<0.001, \mathrm{~N}=9)$, and the QT/QTc interval from $318 \pm 61 / 439 \pm 60 \mathrm{~ms}$ to $483 \pm 86 / 566 \pm 92 \mathrm{~ms}(\mathrm{P}<$ $0.01, \mathrm{~N}=9)$. No significant change was observed in the QRS duration ( $63 \pm 9$ vs $65 \pm 10 \mathrm{~ms}, \mathrm{~N}=9)$.

\section{Bepridil and Vagal Stimulation}

Before the administration of bepridil, VNS-min.V prolonged 


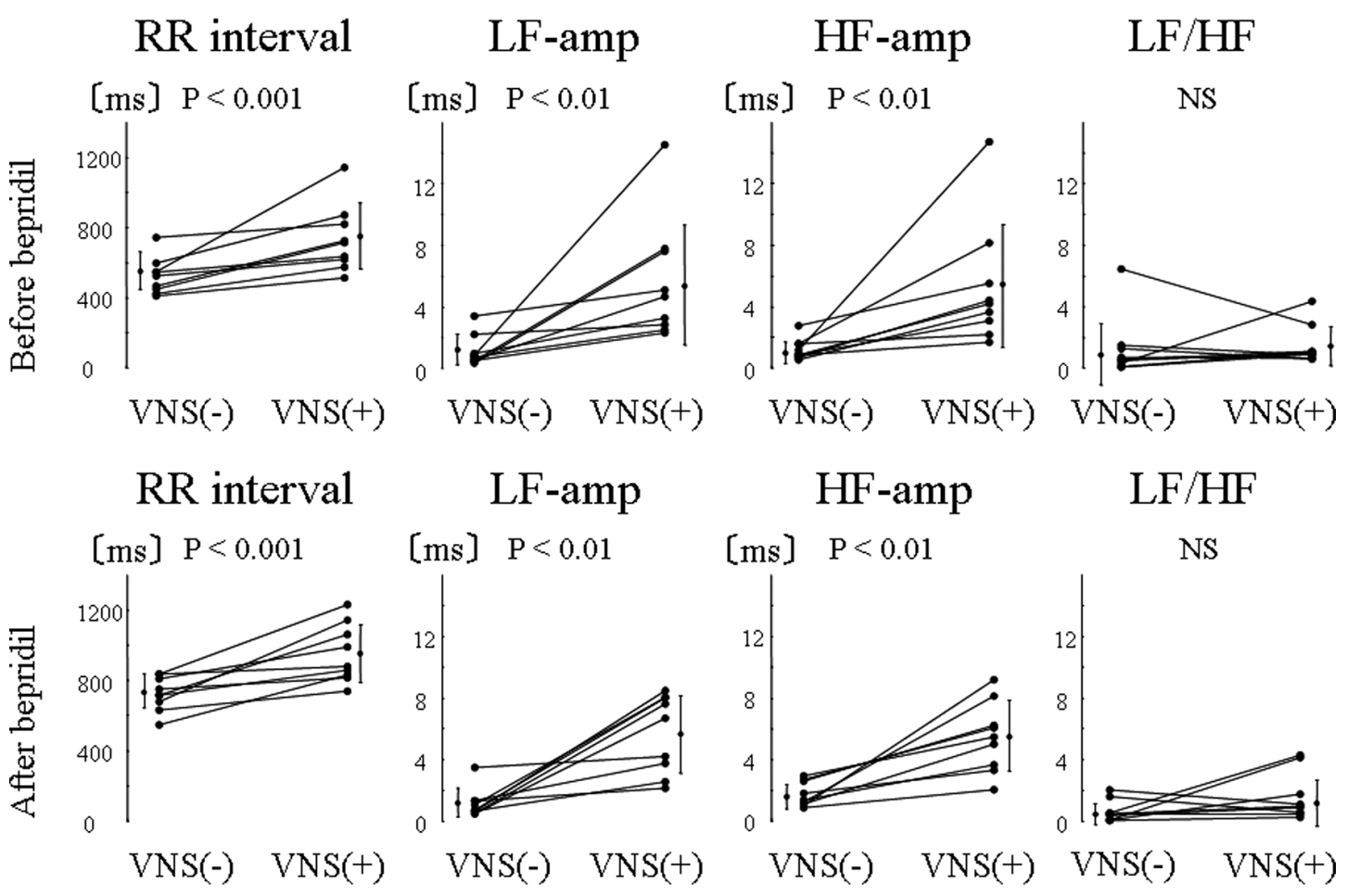

Figure 3. Heart rate variability (HRV) analysis. (Upper) HRV analysis before administration of bepridil. VNS-min.V increased high-frequency (HF)-amp and low-frequency (LF)-amp, although it had no significant effect on LF/HF. (Lower) Similar effects of VNS-min.V were observed after the administration of bepridil. See text for detailed explanations. Abbreviations are as in Figure 1.

the mean sinus cycle length from $523 \pm 104$ to $735 \pm 190 \mathrm{~ms}$ $(\mathrm{P}<0.01, \mathrm{~N}=9)$, and lowered only modestly the systolic/ diastolic blood pressure from $120 \pm 20 / 64 \pm 14$ to $110 \pm 17 / 62 \pm$ $12 \mathrm{mmHg}(\mathrm{NS}, \mathrm{N}=9)$. After the administration of bepridil, VNS-min.V prolonged the mean sinus cycle length from $725 \pm 97$ to $950 \pm 166 \mathrm{~ms}(\mathrm{P}<0.01, \mathrm{~N}=9)$, whereas the mean systolic/diastolic blood pressure remained nearly unchanged, from $101 \pm 18 / 55 \pm 8$ to $104 \pm 14 / 53 \pm 13 \mathrm{mmHg}$ (NS, $\mathrm{N}=9$ ). The mean percentage increase in sinus cycle length induced by VNS-min.V was similar before $(+42 \pm 30 \%)$ vs after $(+32 \pm$ $22 \%$ ) the administration of bepridil ( $\mathrm{NS}, \mathrm{N}=9$ ).

\section{Inducibility of AF}

Before the administration of bepridil, programmed electrical stimulation alone failed to induce AF in all 9 animals. In contrast, sustained AF was induced by a single atrial extrastimulus during the application of VNS-min.V in all 9 experiments (Figure 1A). After the administration of bepridil, AF was non-inducible in all 9 animals before, and was induced in 3 of the 9 experiments during VNS-min.V, representing a $67 \%$ decrease in the inducibility of AF during VNS-min.V $(\mathrm{P}<0.01)$. In the remaining 3 experiments, the mean F-F interval during VNS-min.V increased from $75 \pm 14 \mathrm{~ms}$ before to $147 \pm 13 \mathrm{~ms}$ after the administration of bepridil $(\mathrm{P}<0.05$, $\mathrm{N}=3$; Figure 1B).

\section{Electrophysiological Measurements in the Atria}

At Baseline RA-MAP90 and LA-MAP90 measured 162士 $22(\mathrm{~N}=9)$ and $169 \pm 19 \mathrm{~ms}(\mathrm{~N}=9)$, respectively, ERP-RA measured $133 \pm 23 \mathrm{~ms}(\mathrm{~N}=9)$ and $\mathrm{CT}$-ia $54 \pm 7 \mathrm{~ms}(\mathrm{~N}=9)$ (Table).
VNS-min.V shortened MAP90 by a mean of $-31 \pm 8 \%(\mathrm{~N}=9)$ in the RA and $-22 \pm 12 \%(\mathrm{~N}=9)$ in the LA $(\mathrm{P}<0.01, \mathrm{~N}=9$; Figure 2), and ERP-RA by $-43 \pm 10 \%(\mathrm{P}<0.01, \mathrm{~N}=9$; Table $)$, although it had no significant effect on CT-ia (Table).

After the Administration of Bepridil RA-MAP90, LAMAP90 and ERP-RA duration increased to $185 \pm 33 \mathrm{~ms}(\mathrm{P}<$ $0.05, \mathrm{~N}=9), 190 \pm 15 \mathrm{~ms}(\mathrm{P}<0.05, \mathrm{~N}=9)$ and $180 \pm 19 \mathrm{~ms}(\mathrm{P}<$ 0.001, N=9), respectively (Table). VNS-min.V shortened the RA-MAP90 by $-10 \pm 13 \%$, LA-MAP90 by $-6 \pm 8 \%(\mathrm{~N}=9)$ and ERP-RA by $-16 \pm 11 \%(\mathrm{~N}=9)$. The degree of shortening in these measurements induced by VNS-min.V was significantly smaller $(\mathrm{P}<0.01$ for RA-MAP90 and LA-MAP90, $\mathrm{N}=9$ and $\mathrm{P}<0.001$ for ERP-RA, $\mathrm{N}=9$ ) than before the administration of bepridil. While bepridil prolonged CT-ia from $54 \pm 7$ to $57 \pm 9 \mathrm{~ms}(\mathrm{P}=0.09, \mathrm{~N}=9)$, no further significant change in CT-ia was observed after its administration during VNSmin.V ( $57 \pm 9 \mathrm{~ms}$ vs $57 \pm 10 \mathrm{~ms}, \mathrm{~N}=9$ ).

\section{HRV}

Before the Administration of Bepridil VNS-min.V increased HF-amp from $1.2 \pm 0.7$ to $5.3 \pm 4.0 \mathrm{~ms}(\mathrm{P}<0.01, \mathrm{~N}=9)$ and LF-amp from $1.1 \pm 1.0$ to $5.6 \pm 3.9 \mathrm{~ms}(\mathrm{P}<0.01, \mathrm{~N}=9)$, while the $\mathrm{LF} / \mathrm{HF}$ ratio increased non-significantly from $1.3 \pm 2.0$ to 1.5 $\pm 1.3(\mathrm{~N}=9)$ (Figure 3).

After the Administration of Bepridil No significant effect was observed on LF-amp (from 1.1 \pm 1.0 to $1.1 \pm 0.9 \mathrm{~ms}, \mathrm{~N}=9$ ), HF-amp (from $1.2 \pm 0.7$ to $1.7 \pm 0.8 \mathrm{~ms}, \mathrm{~N}=9$ ), or $\mathrm{LF} / \mathrm{HF}$ ratio (from 1.3 \pm 2.0 to $0.6 \pm 0.7, \mathrm{~N}=9$ ) before VNS-min.V. During VNS-min.V HF-amp increased from $1.7 \pm 0.8$ to $5.4 \pm 2.3 \mathrm{~ms}$ $(\mathrm{P}<0.01, \mathrm{~N}=9)$, and LF-amp from $1.1 \pm 0.9$ to $5.7 \pm 2.5 \mathrm{~ms}(\mathrm{P}<$ 
$0.01, \mathrm{~N}=9)$, leaving the $\mathrm{LF} / \mathrm{HF}$ ratio nearly unchanged $(0.6 \pm$ 0.7 to $1.6 \pm 1.5, \mathrm{~N}=9$; Figure 3 ). The mean magnitude of increase in HF-amp and LF-amp induced by VNS-min.V before vs after bepridil $(+393 \%$ vs $+274 \%$ and $810 \%$ vs $+704 \%$, $\mathrm{N}=9$ ) were not statically significant.

\section{Discussion}

The main findings of this study were: (1) bepridil suppressed the induction of AF during VNS-min.V in two-thirds of the animals, (2) bepridil limited the shortening of MAP90 during VNS-min.V in both atria and (3) the lengthening of the sinus cycle length and increase in HF-amp by VNS-min.V were not statically significant before and after the administration of bepridil.

\section{Facilitation of AF Induction by VNS}

Vagal activation is notoriously proarrhythmic in various types of AF. ${ }^{20}$ This effect is attributed to a shortening of the activation wavelength without significant change in conduction velocity, allowing the coexistence of multiple activation wavefronts in the atria. Acetylcholine, released from the parasympathetic nerve terminals, enhances IKAch, while limiting the pacemaker current by activating muscarinic M2 receptors in the atria. The increase in IKAch shortens atrial repolarization and action potential duration, and suppression of the pacemaker current slows the sinus rate. In this study, as in previous studies, ${ }^{11,21}$ the shortening of MAP90 in the atrium by VNS-min.V coincided with the inducibility of AF.

\section{Pharmacological Therapy of AF}

Inhibition of the sodium current $\left(\mathrm{INa}_{\mathrm{Na}}\right.$ and of the rapid $\left(\mathrm{IKr}_{\mathrm{Kr}}\right.$ and slow (IKs) components of the delayed rectifier potassium current with class I or class III antiarrhythmic drugs are typical objectives of pharmacological suppression of AF. However, the efficacy of sodium channel blockers in preventing the initiation of AF is limited, while amiodarone, a multichannel blocker, is more effective in the suppression of AF.22,23

Recent studies have reported a high efficacy of bepridil in the suppression of paroxysmal and persistent AF. ${ }^{24,25}$ As in the case of amiodarone, bepridil blocks multiple myocardial ionic channels. In this study, MAP90 lengthened in both atria after the administration of bepridil, probably by inhibiting IKr, IKs and the ultra rapid potassium current (Ikur). In contrast, CT-ia was unchanged by the administration of bepridil, suggesting that the inhibitory effect of bepridil on INa in the atrium is weaker in the doses used in this study. However, the effects of INa blockade by antiarrhythmic drugs may be use-dependent. Therefore, when we measured CT-ia at a paced cycle length of $300 \mathrm{~ms}$, no apparent inhibitory effect on INa was observed, whereas the F-F interval was lengthened by the administration of bepridil in the 3 animals in whom AF remained inducible after administration of the drug (Figure 1B).

Bepridil prolonged the spontaneous sinus cycle length and slightly decreased the systolic and diastolic blood pressures in absence of VNS-min.V. These effects were probably due to the inhibition of $\mathrm{L}$ and $\mathrm{T}$ type calcium currents ( $\mathrm{ICa}_{\mathrm{C}}$. Conversely, the inhibition of IKAch in the sinus node by bepridil might have a counteractive effect in the prolongation of sinus cycle length to some degree.

\section{Inhibition of IKAch and HRV}

Anticholinergic antiarrhythmic drugs, such as disopyramide and cibenzoline, are effective in the treatment of vagally mediated AF by preventing the shortening of the atrial action potential duration during VNS. ${ }^{26}$ A similar observation was made in this study, although the electrophysiological mechanism appeared different from that associated with anticholinergic drugs. In our previous study, using the same model of AF, the VNS-induced prolongation of sinus cycle length and increase in HF-amp were counteracted by disopyramide, which effectively blocked the activation of muscarinic $\mathrm{M} 2$ receptors in the atrium.

In this study, the muscarinic M2 receptors appeared to be activated by VNS-min.V, as the prolongation of the sinus cycle length by VNS-min.V was statistically similar both before and after administration of bepridil. This impression was somewhat supported by the analysis of HRV, which revealed a similar degree of increase in HF-amp by VNS-min.V, before and after the administration of bepridil. In contrast, the shortening of MAP90 induced by VNS-min.V in both atria was markedly attenuated after the administration of bepridil. Therefore, we think that, as far as this study shows, it seems possible that inhibition of IKAch by bepridil played a part in this antifibrillatory effect. In a previous study using guineapigs, IKAch in atrial myocytes was inhibited by bepridil in a concentration-dependent manner. ${ }^{10}$ This inhibition of IKAch may be associated with the suppression of AF by bepridil. In addition, the statistically insignificant but non-trivial attenuation in the VNS-min.V induced prolongation of the sinus cycle length ( $42 \pm 30 \%$ vs $32 \pm 22 \%, \mathrm{~N}=9)$ and augmentation of the HF-amp $(393 \pm 349 \%$ vs $274 \pm 256 \%, N=9)$ after bepridil administration could be associated with an inhibitory effect of IKAch in the sinus node by bepridil. Such specific electrophysiological effects to the sinus node can affect the results of HRV analysis. Therefore, HRV analysis in such a situation may limit the accuracy of any autonomic nerve activity measurement in regards to the atrial myocardium. ${ }^{27,28}$

As with the other potential mechanisms, prolongation of the baseline MAP90 due to the inhibition of $\mathrm{IKr}$, IKs and/or Ikur could be associated with the cancellation effect of VNSinduced shortening of the MAP90 in the atrium. Furthermore, because bepridil has non-specific calcium channel blocking effects, calcium-mediated release of Ach from the nerve endings may be prevented through the inhibition of N-type calcium channel current to some degree. ${ }^{29}$

\section{Clinical Implications}

The antifibrillatory effects conferred by bepridil in AF induced by VNS suggest that it might be the drug of choice for AF triggered by vagal nerve activation, particularly in patients suffering from disorders such as prostate hypertrophy or glaucoma. Because cholinergic actions of chemicals constrict smooth muscles, antiarrhythmic drugs that include the anti-cholinergic effect are usually unavailable for patients who suffer from prostate hypertrophy and/or glaucoma, etc. This is because anti-cholinergic action inhibits muscle constriction of the urinary bladder and can induce dysuria and constriction of the ciliary muscle and sphincter muscle of the pupil, which can be a cause of elevation of intraocular pressure. Dobrev et al found that IKAch is continuously increased in patients presenting with chronic AF. ${ }^{30}$ Therefore, like several new antiarrhythmics, which inhibit Ikur or atrial specific INa, ${ }^{31,32}$ drugs with IKAch-blocking properties may play an important role in the management of AF.

\section{Study Limitations}

First, we used the right vagal nerve trunk to apply VNS. Because the right atrium is mainly innervated by the right vagal 
nerve, unilateral nerve stimulation may have affected the results of our study. However, the absolute difference in MAP90 between RA and LA was similar before and during VNS-min.V $(25 \pm 18 \mathrm{~ms}$ during and $15 \pm 13 \mathrm{~ms}$ before VNSmin. $\mathrm{V}$, before bepridil, $\mathrm{P}=0.26, \mathrm{~N}=9$ and $32 \pm 31 \mathrm{~ms}$ during and $39 \pm 35 \mathrm{~ms}$ before VNS-min.V, after bepridil, $\mathrm{P}=0.31$, $\mathrm{N}=9$; Table). Compared to some other studies, ${ }^{12,13}$ we used relatively higher $\mathrm{Hz}$ and lower output for the VNS. However, an obvious vagotonic effect was obtained by the stimulation and we have used this protocol in our previous experimental studies. ${ }^{14,15}$ Second, we did not monitor the serum and tissue concentration of bepridil in this study. Although we completed the experimental protocol after administration of bepridil within $30 \mathrm{~min}$, the serum and tissue concentration of bepridil would certainly have altered during this period and, of course, this may have affected the results of the study. However, there was no statistical difference in the following parameters measured in the basic sinus rhythm among the beginning, middle (approximately $15 \mathrm{~min}$ after starting the study protocol of bepridil) and completion period of the experimental protocol of bepridil; basic cycle length (725 \pm 97 , $710 \pm 56,694 \pm 65 \mathrm{~ms}, \mathrm{P}=0.899$ by ANOVA), blood pressure $(101 \pm 18 / 55 \pm 8,96 \pm 8 / 60 \pm 11,98 \pm 8 / 61 \pm 10 \mathrm{mmHg}, \mathrm{P}=0.805$ for systolic and $\mathrm{P}=0.767$ for diastolic blood pressure by ANOVA), PQ interval (131 $\pm 7,128 \pm 15,125 \pm 15 \mathrm{~ms}, \mathrm{P}=0.673$ by ANOVA), width of QRS complex $(65 \pm 10,65 \pm 5,67 \pm 8 \mathrm{~ms}$, $\mathrm{P}=0.392$ by ANOVA), QT interval (483 $\pm 86,477 \pm 57,460 \pm$ $53 \mathrm{~ms}, \mathrm{P}=0.631$ by ANOVA), RA-MAP90 $(218 \pm 23,227 \pm 35$, $220 \pm 20 \mathrm{~ms}, \mathrm{P}=0.484$ by ANOVA) and LA-MAP90 (218 \pm 23 , $223 \pm 21,217 \pm 21 \mathrm{~ms}, \mathrm{P}=0.755$ by ANOVA). Therefore, we think that the electrophysiological effects of bepridil were present during the experimental protocol. Third, it is usually difficult to obtain a stable MAP recording during the whole experimental period. Because we used Franz catheters to record the MAP from the atriums, it may be possible that activation of stretch activated channel, etc could have altered the configuration of MAP and modified the results of measurement. ${ }^{33,34}$ Perhaps, another recording method (eg, plunge electrode) would affect the analysis of the local repolarization in this experimental protocol to a lesser extent. Fourth, in this study, we focused on the inducibility of AF to estimate antiarrhythmic effects of bepridil. However, the duration and spontaneous terminability of AF are also important antiarrhythmic parameters in the treatment of AF. We performed programmed electrical stimulation from the RA appendage, although, in humans, the triggers of AF are known to frequently originate from the pulmonary vein, LA, or both. ${ }^{8,9}$ Furthermore, we used a relatively gentle protocol of $\mathrm{AF}$ induction, which might have amplified the antifibrillatory effects of bepridil. Fifth, the alteration of rate-dependent change in the MAP90 in both atria can be associated with the antifibrillatory effects of bepridil, but this parameter was not assessed in this study. Sixth, we analyzed HRV from 90-s samples of surface ECG. Although similar samples were used in previous studies, ${ }^{16-18}$ 90-s ECG segments might be too short to acquire reliable LF and HF components. Finally, although we administered low doses of sodium thiopental, anesthetic agents might influence the inducibility of AF and HRV analysis.

\section{Conclusions}

In this experimental study, prevention of VNS-induced shortening of the MAP90 in the atrium seems to play some role in the antiarrhythmic effect of bepridil on AF.

\section{References}

1. Anno T, Furuta T, Itoh M, Kodama I, Toyama J, Yamada K. Electromechanical effects of bepridil on rabbit isolated hearts. $\mathrm{Br} J$ Pharmacol 1984; 81: 41-47.

2. Kobayashi S, Reien Y, Ogura T, Saito T, Masuda Y, Nakaya H. Inhibitory effect of bepridil on hKv1.5 channel current: Comparison with amiodarone and E-4031. Eur J Pharmacol 2001; 430: $149-157$.

3. Wang JC, Kiyosue T, Kiriyama K, Arita M. Bepridil differentially inhibits two delayed rectifier $\mathrm{K}(+)$ currents, $\mathrm{I}(\mathrm{Kr})$ and $\mathrm{I}(\mathrm{Ks})$, in guinea-pig ventricular myocytes. Br J Pharmacol 1999; 128: 1733 1738.

4. Fujiki A, Tsuneda T, Sugao M, Mizumaki K, Inoue H. Usefulness and safety of bepridil in converting persistent atrial fibrillation to sinus rhythm. Am J Cardiol 2003; 92: 472-475.

5. Izumi D, Chinushi M, Watanabe H, Washizuka T, Okamura K, Komura S, et al. Bepridil for drug-refractory ventricular tachyarrhythmias. Intern Med 2007; 46: 119-124.

6. Imai S, Saito F, Takase H, Enomoto M, Aoyama H, Yamaji S, et al. Use of bepridil in combination with Ic antiarrhythmic agent in converting persistent atrial fibrillation to sinus rhythm. Circ J 2008; 72: $709-715$.

7. Niwano S, Sasaki T, Kurokawa S, Kiryu M, Fukaya H, Hatakeyama $\mathrm{Y}$, et al. Predicting the efficacy of antiarrhythmic agents for interrupting persistent atrial fibrillation according to spectral analysis of the fibrillation waves on the surface ECG. Circ J 2009; 73: 12101218.

8. Haïssaguerre M, Jaïs P, Shah DC, Takahashi A, Hocini M, Quiniou $\mathrm{G}$, et al. Spontaneous initiation of atrial fibrillation by ectopic beats originating in the pulmonary veins. $N$ Engl J Med 1998; 339: $659-666$.

9. Kumagai K, Ogawa M, Noguchi H, Yasuda T, Nakashima H, Saku K. Electrophysiologic properties of pulmonary veins assessed using a multielectrode basket catheter. J Am Coll Cardiol 2004; 43: $2281-2289$.

10. Hara Y, Nakaya H. SD-3212, a new class I and IV antiarrhythmic drug: A potent inhibitor of the muscarinic acetylcoline-receptoroperated potassium current in guinea-pig atrial calls. Br J Pharmacol 1995; 116: $2750-2756$.

11. Sugiura H, Chinushi M, Komura S, Hirono T, Aizawa Y. Heart rate variability is a useful parameter for evaluation of anticholinergic effect associated with inducibility of atrial fibrillation. Pacing Clin Electrophysiol 2005; 28: $1208-1214$.

12. Wang Z, Pagé P, Nattel S. Mechanism of Flecainide's antiarrhythmic action in experimental atrial fibrillation. Circ Res 1992; 71: $271-287$.

13. Wang J, Bourne GW, Wang Z, Villemaire C, Talajic M, Nattel S. Comparative mechanisms of antiarrhythmic drug action in experimental atrial fibrillation: Importance of use-dependent effects on refractoriness. Circulation 1993; 88: 1030-1044.

14. Chinushi M, Restivo M, Caref EB, El-Sherif N. Electrophysiological basis of arrhythmogenicity of QT/T alternans in the long-QT syndrome: Tridimensional analysis of the kinetics of cardiac repolarization. Circ Res 1998; 83: 614-628.

15. Chinushi M, Hosaka Y, Washizuka T, Furushima H, Aizawa Y. Arrhythmogenesis of $\mathrm{T}$ wave alternans associated with surface QRS complex alternans and the role of ventricular prematurity: Observations from a canine model of LQT3 syndrome. J Cardiovasc Electrophysiol 2002; 13: 599-604.

16. Ohtomo N, Tanaka Y. New method of time series analysis and "MemCalc." In: Saito K, editor. A recent advance in time series analysis by maximum entropy method. Sapporo, Japan: Hokkaido University Press, 1994; 11-29.

17. Sawada Y, Ohtomo N, Tanaka Y, Tanaka G, Yamakoshi K, Terachi $\mathrm{S}$, et al. New technique for time series analysis combining the maximum entropy method and non-linear least squares method: Its value in heart rate variability analysis. Med Biol Eng Comput 1997; 35: $318-322$.

18. Murasato Y, Hirakawa H, Harada Y, Nakamura T, Hayashida Y. Effects of systemic hypoxia on R-R interval and blood pressure variabilities in conscious rats. Am J Physiol 1998; 275: H797-H804.

19. Wijffels MC, Kirchhof CJ, Dorland R, Allessie MA. Atrial fibrillation begets atrial fibrillation: A study in awake chronically instrumented goats. Circulation 1995; 92: 1954-1968.

20. Yamashita T, Murakawa Y, Sezaki K, Inoue M, Hayami N, Shuzui $\mathrm{Y}$, et al. Circadian variation of paroxysmal atrial fibrillation. Circulation 1997; 96: 1537-1541.

21. Wu TJ, Kim YH, Yashima M, Athill CA, Ting CT, Karagueuzian $\mathrm{HS}$, et al. Progressive action potential duration shortening and the 
conversion from atrial flutter to atrial fibrillation in the isolated canine right atrium. $J$ Am Coll Cardiol 2001; 38: 1757-1765.

22. Roy D, Talajic M, Dorian P, Connolly S, Eisenberg MJ, Green $\mathrm{M}$, et al. Amiodarone to prevent recurrence of atrial fibrillation: Canadian Trial of Atrial Fibrillation Investigators. $N$ Engl J Med 2000; 342: 913-920.

23. Singh BN, Singh SN, Reda DJ, Tang XC, Lopez B, Harris CL, et al; Sotalol Amiodarone Atrial Fibrillation Efficacy Trial (SAFET) Investigators. Amiodarone versus sotalol for atrial fibrillation. N Engl J Med 2005; 352: 1861 - 1872.

24. Fujiki A, Sakamoto T, Iwamoto J, Nishida K, Nagasawa $H$, Mizumaki K, et al. Pharmacological cardioversion of persistent atrial fibrillation with and without a history of drug-resistant paroxysmal atrial fibrillation. Circ J 2006; 70: 1138-1141.

25. Nakazato Y, Yasuda M, Sasaki A, Iida Y, Kawano Y, Nakazato K, et al. Conversion and maintenance of sinus rhythm by bepridil in patients with persistent atrial fibrillation. Circ J 2005; 69: 44-48.

26. Camm J, Ward D, Spurrell RA. The effect of intravenous disopyramide phosphate on recurrent paroxysmal tachycardias. Br J Clin Pharmacol 1979; 8: $441-449$.

27. Miyakoshi M, Ikeda T, Miwa Y, Sakaki K, Ishiguro H, Abe A, et al. Quantitative assessment of cibenzoline administration for vagally mediated paroxysmal atrial fibrillation using frequency-domain heart rate variability analysis. J Cardiol 2009; 54: 86-92.

28. Dias da Silva Valdo J, Viana Públio CC, de Melo Alves R, Fazan
R Jr, Ruscone TG, Porta A, et al. Intravenous amiodarone modifies autonomic balance and increases baroreflex sensitivity in conscious rats. Auton Neurosci 2002; 95: 88-96.

29. Bezprozvanny I, Tsien RW. Voltage-dependent blockade of diverse types of voltage-gated $\mathrm{Ca}^{2+}$ channels expressed in Xenopus oocytes by the $\mathrm{Ca}^{2+}$ channel antagonist mibefradil. Mol Pharmacol 1995; 48: $540-549$.

30. Dobrev D, Friedrich A, Voigt N, Jost N, Wettwer E, Christ T, et al. The $\mathrm{G}$ protein-gated potassium current $\mathrm{I}(\mathrm{K}, \mathrm{ACh})$ is constitutively active in patients with chronic atrial fibrillation. Circulation 2005; 112: 3697-3706.

31. de Haan S, Greiser M, Harks E, Blaauw Y, van Hunnik A, Verheule $\mathrm{S}$, et al. AVE0118, blocker of the transient outward current (I(to)) and ultrarapid delayed rectifier current (I(Kur)), fully restores atrial contractility after cardioversion of atrial fibrillation in the goat. Circulation 2006; 114: 1234-1242.

32. Burashnikov A, Di Diego JM, Zygmunt AC, Belardinelli L, Antzelevitch C. Atrial-selective sodium channel block as a strategy for suppression of atrial fibrillation. Ann N Y Acad Sci 2008; 1123: $105-112$.

33. Ravelli F, Allessie M. Effects of atrial dilatation on refractory period and vulnerability to atrial fibrillation in the isolated Langendorffperfused rabbit heart. Circulation 1997; 96: 1686-1695.

34. Moore HJ, Franz MR. Monophasic action potential recordings in humans. J Cardiovasc Electrophysiol 2007; 18: 787-790. 